\title{
Effect of cell size in calculating frequencies of magnetic modes using micromagnetics: Special role of the uniform mode
}

\author{
R. E. Camley, B. V. McGrath, Y. Khivintsev, and Z. Celinski \\ Center for Magnetism and Magnetic Nanostructures, University of Colorado at Colorado Springs, Colorado 80918, USA \\ Roman Adam and Claus M. Schneider \\ Institute of Solid State Research, Research Center Jülich, D-52425 Jülich, Germany \\ M. Grimsditch \\ Materials Science Division, Argonne National Laboratory, Argonne, Illinois 60439, USA
}

(Received 30 January 2008; published 23 July 2008)

\begin{abstract}
Using the time evolution of the dynamic magnetization, we study the frequencies of spin excitations in small ferromagnetic bars with particular attention to the uniform mode. The calculation is done using micromagnetics, and the frequency of various modes is found as a function of the cell size used in the calculation. We find that the uniform mode and some standing spin-wave modes are relatively insensitive to changes in the cell size. In contrast, edge modes and corner modes show significant frequency changes as the cell size is changed in the micromagnetics calculation. These results justify the use of micromagnetics with larger cells to estimate the frequency of the uniform mode of micronsized magnetic bars. A comparison of experiment and theory shows that the micromagnetics estimate is significantly better than the usual Kittel formula with effective demagnetizing factors; however appreciable differences remain.
\end{abstract}

DOI: 10.1103/PhysRevB.78.024425

PACS number(s): 76.50.+g, 75.30.Ds, 75.75.+a

\section{INTRODUCTION}

One of the standard techniques used in characterizing magnetic materials is ferromagnetic resonance. This method measures the response of a magnetic structure to an externally applied microwave field. Typically this technique couples most strongly to the "uniform" mode, although it is sometimes possible to obtain information on other magnetic excitations in a given structure. ${ }^{1,2}$ The simplest description of the uniform mode is obtained by analogy with a threedimensional (3D) ellipsoid for which the precessions of all spins are in phase and are equal in amplitude; i.e., the magnetization precesses rigidly, with no spatial variation in the dynamic magnetization. In this case, there are demagnetizing fields which come from effective magnetic charges on the surface of the sample. For a 3D ellipsoid, the uniform mode frequency is given by what is often known as the Kittel formula, ${ }^{3}$

$$
f=\gamma \sqrt{\left[H+4 \pi\left(N_{x}-N_{z}\right) M\right]\left[H+4 \pi\left(N_{y}-N_{z}\right) M\right]},
$$

where $\gamma$ is the gyromagnetic ratio, $H$ is the applied field in the $z$ direction, $M$ is the saturation magnetization, and $N_{x}, N_{y}$, and $N_{z}$ are the demagnetizing factors of the $3 \mathrm{D}$ ellipsoid. For a rectangular prism, the effective demagnetizing factors may be approximated by the method given by Aharoni. ${ }^{4}$

More accurate results can be obtained by relaxing the assumptions inherent in obtaining Eq. (1), viz., that the static magnetization and dynamic magnetizations are uniform throughout the sample. There have been some recent elegant theoretical treatments ${ }^{5-7}$ of magnetic excitations in nanostructures which show deviations from Eq. (1). However, some of these techniques still assume that the static magnetization is uniform throughout the sample despite the fact that it is well known that the microscopic magnetic structure may not be uniform and, in small external fields, may even break up into magnetic domains.

One technique for the calculation of the magnetic structure of nano- and microsized objects is to use micromagnetics. These calculations break the structure into small cells. Each cell represents a group of spins which all point in approximately the same direction. The cells interact with each other via dipolar fields and renormalized exchange fields. One often has external fields and anisotropy fields which act on each cell as well. The motion of a the magnetization in a cell is given by the Landau-Lifshitz equation,

$$
\frac{\partial \vec{M}}{\partial t}=-\frac{|\gamma|}{1+\alpha^{2}}\left[\vec{M} \times \vec{H}_{\mathrm{eff}}-\frac{\alpha}{M} \vec{M} \times\left(\vec{M} \times \vec{H}_{\mathrm{eff}}\right)\right],
$$

where $H_{\text {eff }}$ is the effective field acting on a cell, $\vec{M}$ is the magnetization, and $\alpha$ is a dimensionless damping constant. For an equilibrium structure, one looks for a final state where $\vec{M} \times \vec{H}_{\text {eff }}$ is close to zero for all cells. Since the size of the unit cell can play a critical role, it is generally accepted that the cell size must be smaller than the exchange length, typically 5-10 nm for ferromagnetic metals.

As indicated in Eq. (2), micromagnetics can also be used to obtain the dynamic motion of a small object. ${ }^{8-10}$ Although these techniques have yielded results that are in agreement with experimental findings, the role of the elementary cell size in dynamical calculations has not been suitably addressed. However, if the cell dimension (5-10 nm) restriction is enforced, the total number of cells required for the simulation of the normal modes of particles that are larger than about $1 \mu \mathrm{m}$ is so large that it essentially precludes any simulation. Here we wish to determine if there are any cir- 
cumstances where the $5-10 \mathrm{~nm}$ cell size restriction relevant for the calculation of the static structure can be relaxed in finding the dynamic modes. The question is one of considerable practical importance since many device situations include magnetic structures at or above the $1 \mu \mathrm{m}$ scale. One indication that it might be possible to find situations where the size restriction might be relaxed is found in analytic theories for the modes in nanosized objects. As we have noted, such theories generally begin with an assumption of a uniform static magnetization throughout the nano-object. In Ref. 8 the effect of cell size was also briefly addressed and it was found that some of the modes depended more strongly than others on the cell size. Here we address this issue more systematically.

In this paper, we use a dynamical micromagnetic scheme to calculate the static and dynamic behaviors of nanosized rectangular bars. We examine how the dynamical frequencies of the different modes depend on the cell size used in the calculation. Many modes show substantial dependence on the cell size until the size is brought into the 5-10 $\mathrm{nm}$ range. However the uniform mode or "ferromagnetic resonance" mode shows a remarkable independence from the cell dimensions. Indeed for this mode calculations with $6 \mathrm{~nm}$ cells give essentially the same result as with $100 \mathrm{~nm}$ cells.

The findings reported here will be quite helpful because they allow one to use micromagnetic calculations as an approximation on larger, micronsized bars without having so many cells that the calculation becomes impossible. As an example, we compare theory and measurements for the uniform mode in Permalloy bars which are $4 \mu \mathrm{m}$ wide, $8 \mu \mathrm{m}$ long, and $100 \mathrm{~nm}$ thick. We use cell sizes of $200 \times 200$ $\times 100 \mathrm{~nm}^{3}$. However, despite these large cells, the micromagnetics calculations are significantly closer to the experimental data than the Kittel approximation based on a uniform magnetization.

\section{CALCULATIONAL METHOD}

We use a variation of the method outlined in Ref. 8. The motion of the magnetization in each cell is governed by the Landau-Lifshitz equation [Eq. (2)]. The effective field acting on a cell is given by a sum of the dipole fields, effective exchange fields and the applied field:

$$
\vec{H}_{\text {eff }}=\vec{H}_{\text {dipolar }}+\vec{H}_{\text {exchange }}+\vec{H}_{\text {applied }} .
$$

In this work we neglect crystalline anisotropy, but this can be easily included. The external and constant field $\vec{H}_{\text {applied }}$ is directed in the plane of the sample, either parallel or perpendicular to the long axis of the bar. The dipolar terms are found using the Newell tensor ${ }^{11} \stackrel{\leftrightarrow}{N}$. The dipole field acting on a cell $i$ is given by a sum over all the cells $j$, including cell $i$ :

$$
\vec{H}_{\text {dipolar }-i}=-\sum_{j} \stackrel{\leftrightarrow}{N}\left(r_{i}-r_{j}\right) \cdot \vec{M}_{j}
$$

Here $r_{i}$ is the position vector of the $i$ th cell and $\vec{M}_{j}$ is the magnetization of cell $j$. The exchange fields are given by

$$
\vec{H}_{\text {exchange }}=J \sum_{n n} \vec{m}_{n n}
$$

The sum is over the nearest neighbors of the cell of interest, and $\vec{m}_{n n}$ is a unit vector indicating the direction of the magnetization in the nearest-neighbor cells. $J$ is the effective exchange coupling constant which depends on the cell size:

$$
J=\frac{2 A}{d^{2} M} 10^{8}
$$

where $A$ is the usual exchange coupling constant measured in ergs $/ \mathrm{cm}, d$ is the side length of the cell in the plane in nanometers, and $M$ is the saturation magnetization in kiloGauss. The units of $J$ are kiloGauss.

To find the equilibrium state of the object, one starts the system in an arbitrary configuration and iterates forward in time using a numerical method such as the fourth-order Runge-Kutta scheme. Eventually the torque on each cell is nearly zero, and the system is in an equilibrium state. We have checked that our results are equivalent to those found by OOMMF. ${ }^{12}$ Once in equilibrium, the magnetization in each cell is then given a small perturbation and the time evolution of the system as a whole and of each cell is recorded. From the Fourier transform of the time evolution, one can obtain the eigenfrequencies of the modes which are excited by a particular perturbation.

\section{BEHAVIOR OF MODES AS A FUNCTION OF CELL SIZE}

In order to understand how cell size in micromagnetics influences the frequencies of the individual modes, we study a small magnetic parallelepiped that is $6 \times 240 \times 480 \mathrm{~nm}^{3}$. In this structure the lateral cell size can easily be varied from 6 to $60 \mathrm{~nm}$. We use $\gamma=2.9 \mathrm{GHz} / \mathrm{kOe}$ and $M=0.7639 \mathrm{kG}$, which will match with the experimental parameters for Permalloy in Sec. IV. The applied field is $2 \mathrm{kOe}$.

In order to have a qualitative feel for the modes which appear in the dynamic micromagnetic calculation, we present the results for three modes that represent the major types of modes that are encountered in rectangular particles. The calculations are done for the case where $H$ is in plane and perpendicular to the long axis and $H=2 \mathrm{kOe}$. We chose this value of the field because it is large enough to lead to an almost saturated state. The lateral cell dimensions are 20 $\times 20 \mathrm{~nm}^{2}$. The amplitude distribution within the particle is shown in Fig. 1 for three modes. Similar results are found for calculations with different cell dimensions. These schematics show the out-of-plane amplitudes at a given instant; i.e., it is a snapshot of the motion of the magnetization. The lowest frequency mode is an edge mode (a) where the excitation is localized at the edges of the sample. The uniform mode (b) has all spins pointing out of plane at the same time. The amplitude is largest near the center of the sample, but near the ends the spins are effectively pinned and the amplitude is near zero. The standing-wave-like solutions (c) have some regions where the spins are up and other regions where they are down.

Figure 2 presents the frequencies of four modes as a function of cell size for the case where the field is parallel to the 
a)

b)
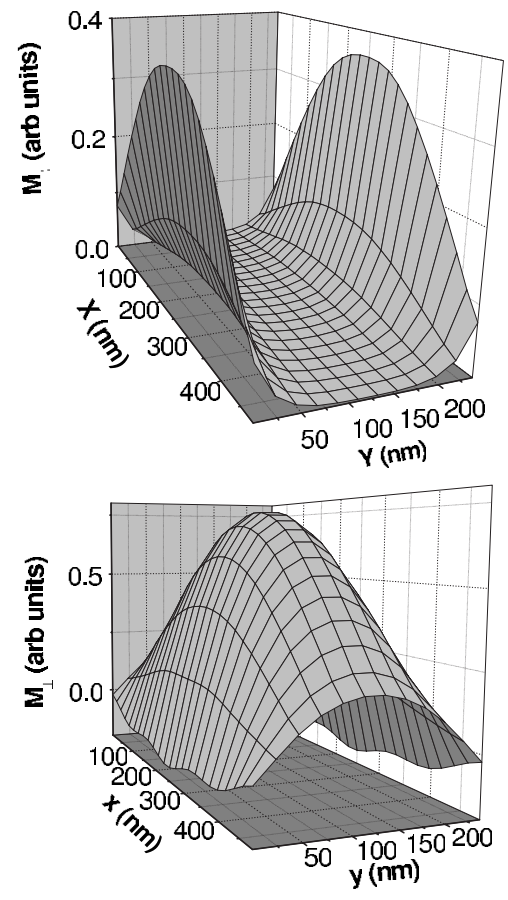

c)

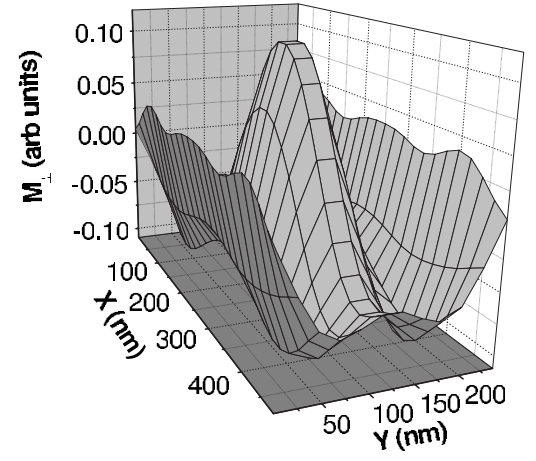

FIG. 1. Typical modes in rectangular ferromagnetic bars. (a) shows a snapshot the out-of-plane magnetization for an edge mode at a frequency of $9.76 \mathrm{GHz}$. (b) shows the uniform mode at 13.76 $\mathrm{GHz}$, and (c) shows a standing-wave mode at $15.1 \mathrm{GHz}$. The applied field, $H=2 \mathrm{kOe}$, is in the plane of the sample and along the short axis.

long axis of the bars. The primary observations from this graph are that the edge modes and corner modes are quite sensitive to the cell size, the standing-wave mode is slightly sensitive to the cell size, and the uniform mode is nearly independent of the cell size. Nonetheless, the frequency for the uniform mode is different from that found with the Kittel formula. The demagnetizing factors used in the Kittel formula ${ }^{4}$ are $N_{x}=0.9437, N_{y}=0.0378$, and $N_{z}=0.0185$; and the uniform mode frequency from Eq. (1) is $14.15 \mathrm{GHz}$.

Figure 3 presents the frequencies of the observed modes as a function of cell size for the case where the $2 \mathrm{kG}$ field is applied in plane but perpendicular to the long axis of the bars. Again we note that the uniform mode and standingwave mode are nearly independent of the cell size, but that the corner and edge modes show a strong dependence on the size of the cell. Note that the frequency of the uniform mode

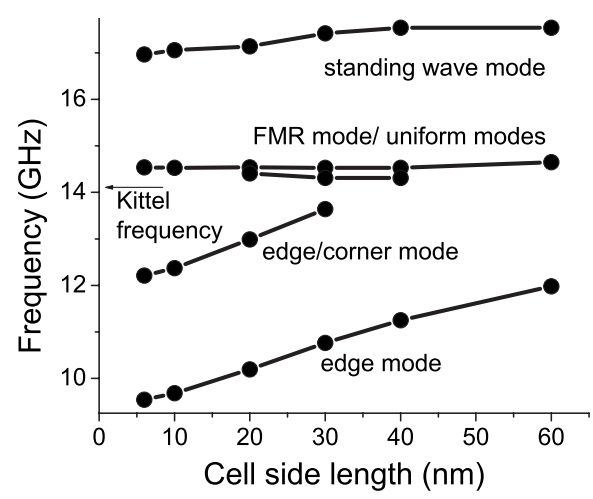

FIG. 2. Frequency of excitations calculated for a $6 \times 240$ $\times 480 \mathrm{~nm}^{3}$ rectangular Permalloy bar as a function of the side length $d$ of the cell. The cells are $6 \mathrm{~nm} \times d \times d$ in size, and an applied field of $2 \mathrm{kOe}$ is directed along the long axis. The double line of data for the uniform mode occurs when there is a hybridization of the uniform mode with two other modes.

obtained from the Kittel formula is substantially below that found in the micromagnetics calculation.

There are two questions that need to be answered: (1) Why are the frequencies of the uniform and standing-wave modes so much less dependent on cell size than the edge or corner modes? (2) Why is the frequency found by micromagnetics different from that found by the Kittel formula and which one is correct?

We believe that both effects are related to the nonuniformity of the demagnetizing fields. In rectangular particles the demagnetizing fields (both static and dynamic) are highly nonuniform. One effect of these nonuniformities results in changes in the mode profiles that lead to pinninglike effects. It has been noted by several groups that the uniform mode is nearly pinned at the edges of the structure; i.e., the amplitude of the dynamic motion is significantly reduced at the edges compared to the center of the structure. When the cell sizes are changed, it is the spin configuration near the edges that usually shows the largest changes. However, because the uniform mode is dynamically pinned near the edges, these changes in static structure do not produce changes in the

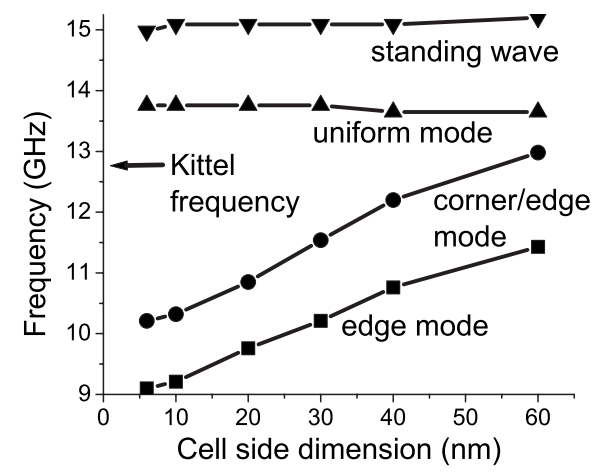

FIG. 3. Frequency of excitations calculated for a $6 \times 240$ $\times 480 \mathrm{~nm}^{3}$ rectangular Permalloy bar as a function of the side length $d$ of the cell. The cells are $6 \mathrm{~nm} \times d \times d$ in size, and an applied field of $2 \mathrm{kOe}$ is directed perpendicular to the long axis along the $240 \mathrm{~nm}$ axis. 
dynamical uniform mode. In contrast, the edge modes and corner modes are strongly localized near the edges of the sample and as a result show significant changes as the cell size changes.

Since the Kittel formulation is basically equivalent to a micromagnetic simulation with a single cell and thereby does not allow for any degree of pinning at the ends, it also becomes clear why the Kittel formula might not lead to the correct result. However, it is less obvious exactly how the micromagnetics result will change the frequency. In the examples above, one finds that the Kittel frequency lies below that found by micromagnetics. We note that for the case shown in Fig. 3, the uniform mode remains nearly constant with frequency until the cell size is about $200 \mathrm{~nm}$ and the total number of cells is below 10 . For a very small number of cells, one recovers the Kittel result.

An additional question relates to the cell size that is necessary for a proper description of the edge and corner modes. One could expect that for these modes one has to have a proper description of the static configuration near the edges. Thus the cell size would need to be on the order of the exchange length, say 2-3 nm. Such calculations could be prohibitive in terms of time even for this relatively small structure. We estimate that adding another point in Figs. 2 and 3 at a cell size of $3 \mathrm{~nm}$ could take calculations of up to 60 days with our computing facilities. Nonetheless, projecting the curves of Figs. 2 and 3 to smaller cell sizes allows one to make reasonable estimates for the values and errors associated with all the modes. In fact, one can already see a slight flattening in Figs. 2 and 3 for the edge and corner mode frequencies at the $6 \mathrm{~nm}$ cell size. This is consistent with the idea that a cell size of $2-3 \mathrm{~nm}$ might be sufficient to properly describe the edge and corner modes.

\section{COMPARISON OF THEORY AND EXPERIMENT}

There have been several recent reports on the response of micronsized magnetic bars to microwave fields. ${ }^{2,13,14}$ It is clear in these studies that the main absorption occurs at the frequency of the uniform mode. In this section we present a careful comparison of some experimental results on Permalloy bars with different theoretical approaches. We examined a number of Permalloy bars of different sizes, but we will concentrate on bars which are $8 \mu \mathrm{m}$ long, $4 \mu \mathrm{m}$ wide, and $100 \mathrm{~nm}$ thick.

Using a coplanar waveguide and a network analyzer, ${ }^{2}$ we have made measurements on the absorption of the microwave energy by the Permalloy (Py) bars as a function of frequency for different magnetic fields. Two geometries were investigated: (1) the static magnetic field is parallel to the long axis of a Permalloy bar, and (2) the static magnetic field is perpendicular to the long axis but still in the plane of the sample. In Fig. 4(a) we present the frequency of the main absorption peak as a function of magnetic field for the two geometries (filled circles for $H$ parallel to the long axis and open circles for $H$ parallel to the short axis). We also show a theoretical fitting (full lines) based on the Kittel formula. [The demagnetizing factors in Eq. (1) are $N_{x}=0.944 N_{y}$ $=0.0378$ and $N_{z}=0.0184$ for the case that the external field is

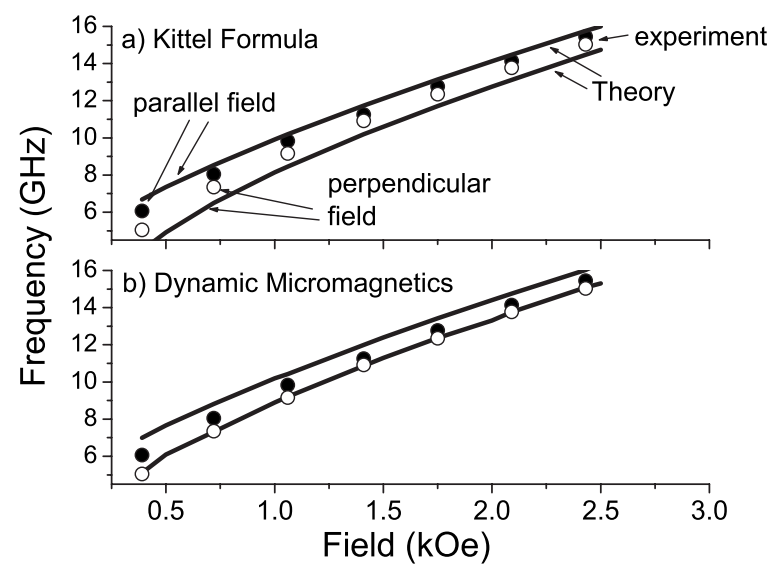

FIG. 4. Experimental and theoretical results for frequency of the uniform mode as a function of external field in a $100 \mathrm{~nm}$ $\times 4 \mu \mathrm{m} \times 8 \mu \mathrm{m}$ Py bar. The dots represent the experimental data; the lines are the theoretical calculations. Closed circles are for the field parallel to the long axis; open circles are for the field perpendicular to the long axis but in the plane of the sample. The theoretical results, calculated using the Kittel formula, shown in (a) provide a poorer description of the experimental results than those in (b), which were obtained using micromagnetics.

along the long axis. When the field is perpendicular to the long axis, the demagnetizing factors in Eq. (1) are $N_{x}$ $=0.944, N_{y}=0.0184$, and $N_{z}=0.0378$. $]$ It is immediately obvious that the experimental data show a much narrower separation in frequency between the two geometries than that which is predicted by the theoretical results.

An important question is whether these differences could be caused by variations in the experimental conditions and/or material parameters. To decrease the difference in frequency, one needs to reduce the effect of the demagnetizing fields. This could be done either by varying the particle shape slightly or by reducing $M$. We find that if the thickness of the bar is reduced by $10 \%$, there are only minimal changes in the demagnetizing factors and that the resulting frequencies are also minimally changed. A similar behavior is found if the length or the width is changed by about $10 \%$. Because errors in size can be controlled to better than $10 \%$, we can conclude that geometrical factors cannot reconcile theory and experiment. Similarly, if $M$ were to be reduced sufficiently to narrow the difference in frequency between the two geometries, the data points would then lie well above the theoretical curves. So, changes in $M$ can also be discarded as an explanation of the differences.

In Fig. 4(b), we compare experiment with the results of micromagnetics calculations. For these simulations, the cell size is $200 \times 200 \times 100 \mathrm{~nm}^{3}$. We use 40 cells for the length, 20 cells for the width, and 1 cell for the thickness. The total time of the simulation is sufficiently long so as to lead to a frequency resolution of under $0.1 \mathrm{GHz}$ after the Fourier transform. The simulations provide a better fit to the experimental data than that obtained using the Kittel formula. In order to see this more clearly, Fig. 5 shows the differences in frequency between the external field applied along the long axis ( $8 \mu \mathrm{m}$ length) and along the short axis ( $4 \mu \mathrm{m}$ length.) We attribute the improvement of the simulations over the 


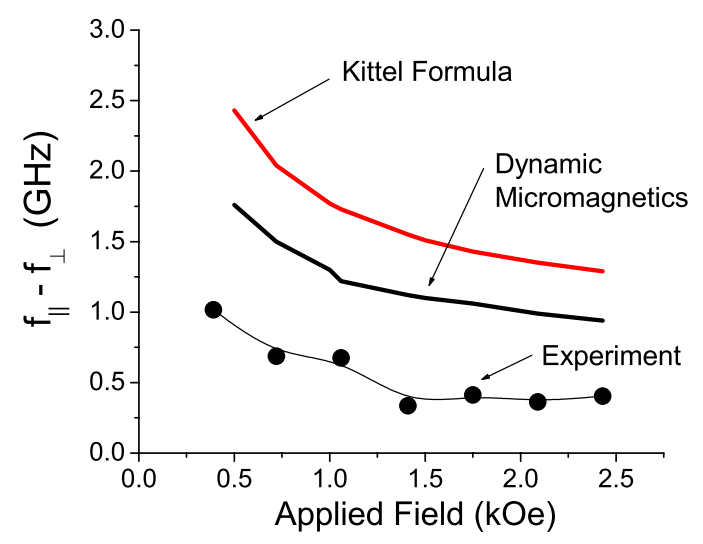

FIG. 5. (Color online) A comparison of the difference in frequency between the cases where the magnetic field is applied along the long axis $(8 \mu \mathrm{m})$ and along the short axis $(4 \mu \mathrm{m})$. The dynamic micromagnetic calculation reduces the error substantially compared to the error from the Kittel formula.

Kittel formula to that fact that the assumptions of the Kittel formula, a rigid precession of all spins, are not imposed in the simulations.

\section{SUMMARY AND DISCUSSION}

We have explored the variation of the frequencies of excitations in small ferromagnetic bars as a function of cell size. We find that the uniform mode and some standing spinwave modes are relatively insensitive to changes in cell size. In contrast, edge modes and corner modes show significant frequency changes as the cell size is changed in the micromagnetics calculation.

All of the calculations presented here are for states where the bar is close to saturation. As mentioned earlier, the assumption of saturation is used in analytic calculations for long bars and the results here serve as some support for those calculations. Clearly if the bar is broken into domains, then micromagnetics may still be used to obtain the dynamical modes, but one must use smaller cells.

Although Fig. 5 clearly shows that simulations provide a more accurate description of the frequency of the uniform precession mode than that by the Kittel formula, it still leaves unanswered the question of why there is a discrepancy between the simulations and the experiments. This is bothersome since we argued that there are no simple parameters that can be adjusted to improve agreement. We speculate that the additional discrepancies might be due to the fact that magnetization of the real sample is not fully saturated or that the edges of the structures are significantly rounded. At this stage we leave it as an open question. Nonetheless, the main point remains unchanged-that dynamic micromagnetics, even with large cells, can provide a better approximation for the frequency of the uniform mode in large bars than does the Kittel approximation.

We have carried out similar calculations for the frequency of the uniform mode in vortex structures ${ }^{15-17}$ in square particles with surprisingly similar results. We studied the frequencies of a $20 \times 300 \times 300 \mathrm{~nm}^{3}$ Co nanostructure in zero applied field. $A$ variation of the side length of the cell from $d=10 \mathrm{~nm}$ to $d=30 \mathrm{~nm}$ results in a frequency shift of less than $1 \%$ for the uniform mode. Varying $d$ from 10 to $60 \mathrm{~nm}$ gives a frequency shift of about $4 \%$. In contrast, a corner mode frequency varies by about $11 \%$ in changing from $d$ $=10 \mathrm{~nm}$ to $d=30 \mathrm{~nm}$. Thus even in a vortex state the uniform mode is relatively insensitive to the detailed structure of the static magnetization.

\section{ACKNOWLEDGMENT}

This work was supported by DOA (Grant No. W911NF04-1-0247).
${ }^{1}$ C. Bayer, J. Jorzick, B. Hillebrands, S. O. Demokritov, R. Kouba, R. Bozinoski, A. N. Slavin, K. Y. Guslienko, D. V. Berkov, N. L. Gorn, and M. P. Kostylev, Phys. Rev. B 72, 064427 (2005).

${ }^{2}$ Roman Adam, Yuri Khivintsev, Riccardo Hertel, Claus M. Schneider, Andrew Hutchison, Robert Camley, and Zbigniew Celinski, J. Appl. Phys. 101, 09F516 (2007).

${ }^{3}$ Charles Kittel, Phys. Rev. 73, 155 (1948).

${ }^{4}$ A. Aharoni, J. Appl. Phys. 83, 3432 (1998).

${ }^{5}$ Rodrigo Arias and D. L. Mills, Phys. Rev. B 70, 094414 (2004).

${ }^{6}$ Rodrigo Arias and D. L. Mills, Phys. Rev. B 72, 104418 (2005).

${ }^{7}$ K. Yu. Guslienko, S. O. Demokritov, B. Hillebrands, and A. N. Slavin, Phys. Rev. B 66, 132402 (2002).

${ }^{8}$ M. Grimsditch, G. K. Leaf, H. G. Kaper, D. A. Karpeev, and R. E. Camley, Phys. Rev. B 69, 174428 (2004).

${ }^{9}$ R. D. McMichael and M. D. Stiles, J. Appl. Phys. 97, 10J901 (2005).

${ }^{10}$ Xiaobin Zhu, Zhigang Liu, Vitali Metlushko, L. Giovannini, F. Montoncello, F. Nizzoli, and Mark R. Freeman, J. Appl. Phys.
99, 08F307 (2006).

${ }^{11}$ Andrew J. Newell, Wyn Williams, and David J. Dunlop, J. Geophys. Res. 98, 9551 (1993).

${ }^{12}$ M. Donahue and D. Porter, “OOMMF User's Guide, Version 1.0," National Institute of Standards and Technology Interagency Report No. NISTIR 6376, 1999, URL: http:// math.nist.gov/oommf.

${ }^{13}$ M. Grimsditch, F. Y. Fradin, Y. Ji, A. Hoffmann, R. E. Camley, V. Metlushko, and V. Novosad, Phys. Rev. Lett. 96, 047401 (2006).

${ }^{14}$ R. Meckenstock, I. Barsukov, C. Bircan, A. Remhoff, D. Dietzel, and D. Spoddig J. Appl. Phys. 99, 08 C706 (2006).

${ }^{15}$ M. Yan, G. Leaf, H. Kaper, R. Camley, and M. Grimsditch, Phys. Rev. B 73, 014425 (2006).

${ }^{16}$ K. Perzlmaier, M. Buess, C. H. Back, V. E. Demidov, B. Hillebrands, and S. O. Demokritov, Phys. Rev. Lett. 94, 057202 (2005).

${ }^{17}$ J. Raabe, C. Quitmann, C. H. Back, F. Nolting, S. Johnson, and C. Buehler, Phys. Rev. Lett. 94, 217204 (2005). 